\title{
Dynamic contrast-enhanced breast MRI features correlate with invasive breast cancer angiogenesis
}

\author{
Jennifer Xiao ${ }^{1}$, Habib Rahbar $\mathbb{D}^{1,2}$, Daniel S. Hippe $\mathbb{D}^{1}$, Mara H. Rendi ${ }^{3}$, Elizabeth U. Parker ${ }^{3}$, Neal Shekar (iD) ${ }^{1}$ Michael Hirano ${ }^{1}$,
} Kevin J. Cheung ${ }^{4,5}$ and Savannah C. Partridge $\mathbb{D}^{1,2 \infty}$

Angiogenesis is a critical component of breast cancer development, and identification of imaging-based angiogenesis assays has prognostic and treatment implications. We evaluated the association of semi-quantitative kinetic and radiomic breast cancer features on dynamic contrast-enhanced (DCE)-MRI with microvessel density (MVD), a marker for angiogenesis. Invasive breast cancer kinetic features (initial peak percent enhancement [PE], signal enhancement ratio [SER], functional tumor volume [FTV], and washout fraction [WF]), radiomics features (108 total features reflecting tumor morphology, signal intensity, and texture), and MVD (by histologic CD31 immunostaining) were measured in 27 patients (1/2016-7/2017). Lesions with high MVD levels demonstrated higher peak SER than lesions with low MVD (mean: 1.94 vs. 1.61, area under the receiver operating characteristic curve [AUC] $=0.79$, $p=0.009$ ) and higher WF (mean: $50.6 \%$ vs. $22.5 \%, A U C=0.87, p=0.001$ ). Several radiomics texture features were also promising for predicting increased MVD (maximum AUC $=0.84, p=0.002$ ). Our study suggests DCE-MRI can non-invasively assess breast cancer angiogenesis, which could stratify biology and optimize treatments.

npj Breast Cancer (2021)7:42; https://doi.org/10.1038/s41523-021-00247-3

\section{INTRODUCTION}

Angiogenesis is a critical factor in the development and progression of breast cancers. Once primarily thought to be driven by genetic alterations of ductal epithelial cells, breast cancer tumorigenesis and metastasis are now recognized also to be dependent on microenvironment factors including basement membrane permeability to allow stromal invasion, local immune suppression to facilitate malignant cells evasion, and angiogenesis to supply nutrients for tumor growth and hematogenous spread ${ }^{1}$. Biologically, angiogenesis is initiated by hypoxic insult to the developing malignancy, which triggers activation of hypoxia inducible factor transcriptional activity (e.g. HIF-1), leading to vascular endothelial growth factor (VEGF) production and release ${ }^{2}$. In turn, VEGF initiates a cascade of endothelial cell proliferation, migration, tube formation, and maturation into microvessels ${ }^{3}$. Clinically, higher levels of angiogenesis are associated with worse patient outcomes in breast cancer ${ }^{4-6}$ and the development of distant metastasis ${ }^{7}$, which also suggests treatment possibilities with anti-angiogenic therapies ${ }^{8}$.

As angiogenesis is a dynamic in vivo process, its quantitation pathologically relies on indirect measures, including the surrogate marker of microvessel density (MVD). MVD is measured by counting small and tortuous vessels in the tumor tissue by immunohistochemical staining using antibodies such as factor VIII antigen (von Willebrand factor), CD31 and CD34 4,9,10. High MVD has been shown to predict poor survival in women with invasive breast cancer, with especially high prognostic value in women who present with lymph node negative disease, and has been proposed as a marker to identify patients at high risk of recurrence 4 . High MVD is also associated with other aggressive features, including higher histologic grade and negative estrogen receptor status ${ }^{11,12}$ and has been shown to correlate with the occurrence of osseous metastases ${ }^{13}$. Although MVD assessments vary in technique, studies have shown that inter- and intra-reader variability can be low with appropriate training ${ }^{14}$.

Dynamic contrast-enhanced magnetic resonance imaging (DCE$\mathrm{MRI}$ ) is routinely utilized in patients with newly diagnosed breast cancer to assess the local extent of disease and assist in surgical and treatment planning. DCE-MRI probes the vascular environment over several timepoints after intravenous injection of a contrast agent, and the varying signal can be described using quantitative enhancement kinetic features. During routine interpretation, semi-quantitative enhancement kinetic features are often utilized to improve specificity for identifying malignancy, including initial peak enhancement and the presence of delayed phase washout, as defined by the American College of Radiology (ACR) Breast Imaging-Reporting And Data System (BI-RADS) Breast MRI Atlas ${ }^{15-17}$. These semi-quantitative kinetics features have been hypothesized to reflect tumor angiogenesis ${ }^{9,18,19}$, though there is a paucity of literature supporting this directly. Additionally, recent advances in computational image analysis have given rise to the new field of "radiomics", where a high dimensional panel of quantitative imaging features are extracted from routine diagnostic radiology studies, which further expands the potential to utilize DCE-MRI to non-invasively characterize underlying tumor architecture, biology, and function ${ }^{20,21}$.

Thus, we sought to assess the association of breast DCE-MRI enhancement features with angiogenesis using semi-quantitative kinetic measurements and radiomics analyses. Specifically, we hypothesized that MRI parameters that reflect early delivery of contrast [initial peak percent enhancement (peak PE)], rapid clearance of contrast [peak signal enhancement ratio (peak SER) and washout fraction (WF)], and greater functional tumor volume (FTV) would correlate with higher levels of MVD. As an exploratory aim, we sought to assess the relationships between comprehensive

\footnotetext{
${ }^{1}$ Department of Radiology, University of Washington, Seattle, WA, USA. ${ }^{2}$ Breast Imaging, Seattle Cancer Care Alliance, Seattle, WA, USA. ${ }^{3}$ Department of Pathology, University of Washington, Seattle, WA, USA. ${ }^{4}$ Department of Medicine, Division of Medical Oncology, University of Washington, Seattle, WA, USA. ${ }^{5}$ Translational Research Program, Public Health Sciences and Human Biology Divisions, Fred Hutchinson Cancer Research Center, Seattle, WA, USA. ${ }_{\text {email: scp3@uw.edu }}$
} 
radiomics analysis-based features of intratumoral heterogeneity and texture with MVD.

\section{RESULTS}

\section{Patient cohort and characteristics}

A total of 33 patients with a diagnosis of invasive breast cancer consented to participate in the study and underwent routine DCEMRI assessment as well as additional pathologic assessment of MVD. Six patients were subsequently excluded from the study due to either obscuration of MRI findings by post-biopsy changes ( $n=$ 4) or unavailable pathologic $(n=2)$ specimens, leaving a final cohort of 27 patients (median age $=53$ years, range $=30$ to 88) with complete data. Median tumor size was $23 \mathrm{~mm}$ (range 10-101), with 23 lesions diagnosed as invasive ductal carcinoma (IDC), three invasive lobular carcinoma (ILC), and one invasive mammary carcinoma not otherwise specified. Median MVD was 17 (range 8-56) microvessels per $0.152 \mathrm{~mm}^{2}(\times 400)$ field, with 13 invasive cancers categorized as high MVD and 14 low MVD. Other histopathologic characteristics, including Nottingham Grade, Ki-67, hormone receptor status, human epidermal growth factor 2 (HER2) status, and T stage are summarized in Table 1. None of these patient and tumor characteristics differed significantly between MVD groups ( $p>0.05$ for each) (Supplementary Table 1).

\section{Associations between kinetic parameters and MVD}

Both peak SER and WF kinetic features were positively associated with MVD. Compared with counterparts with low MVD, invasive breast cancers with high MVD exhibited higher peak SER values (median: 1.94 vs. 1.61, area under the receiver operating characteristic curve $[\mathrm{AUC}]=0.79, p=0.009$ ) and higher WF (median: $50.6 \%$ vs. $22.5 \%, \mathrm{AUC}=0.87, p=0.001$ ), which were statistically significant at the Bonferroni-corrected $a=0.0125$ (Fig. 1). However, there was no significant relationship between initial peak PE (median: $214 \%$ vs. $199 \%, A U C=0.61, p=0.35$ ) or FTV (median: 3.0 vs. $4.7 \mathrm{~cm}^{3}, \mathrm{AUC}=0.60, p=0.40$ ) and MVD (Table 2). As a sensitivity analysis, the semi-quantitative kinetic parameters were also compared with continuous MVD counts. Consistent with the primary analysis, peak SER (Spearman's $r=$ $0.55, p=0.003$ ) and WF (Spearman's $r=0.60, p=0.001$ ) were significantly correlated with higher MVD on a continuous basis (Supplementary Table 2). Examples of two lesions with high and low MVD are shown in Fig. 2.

\section{Associations of radiomic analysis with MVD}

Exploratory analysis of the association of radiomic features revealed multiple features to be potentially associated with $M V D$, including several GLDM features, GLCM features and GLRLM features $(p<0.01)$, as shown in Table 3 . Results for all radiomics features are shown in Supplementary Table 3 and Supplementary Table 4. Two texture-based radiomics feature in particular had large estimated associations with MVD: GLCM-ClusterProminence (AUC $=0.84, p=0.002$ ), which measures skewness and asymmetry of the GLCM (a matrix describing the distribution of neighboring voxel gray levels), and GLRLM-LongRunHighGrayLevelEmphasis (AUC $=0.83, p=0.003$ ), which measures the joint distribution of long run lengths with higher gray-level values (Fig. 3). Relative performance of kinetics and radiomics features for differentiating lesions with low versus high MVD (odds ratios and AUCs) is shown in Fig. 4.

\section{DISCUSSION}

In this single-institution study of women diagnosed with invasive breast cancer, we found that tumors with higher MVD exhibited

\begin{tabular}{|c|c|}
\hline Variable & Value \\
\hline Age, years & $53(30-88)$ \\
\hline \multicolumn{2}{|l|}{ Histology } \\
\hline Invasive ductal carcinoma & $23(85)$ \\
\hline Invasive lobular carcinoma & $3(11)$ \\
\hline Invasive mammary carcinoma & $1(4)$ \\
\hline \multicolumn{2}{|l|}{ Nottingham grade } \\
\hline 1 & $7(26)$ \\
\hline 2 & $7(26)$ \\
\hline 3 & $13(38)$ \\
\hline \multicolumn{2}{|l|}{ Ki-67 } \\
\hline High & $14(52)$ \\
\hline Low & $9(33)$ \\
\hline Not available & $4(15)$ \\
\hline \multicolumn{2}{|l|}{ Estrogen receptor } \\
\hline Positive & $20(74)$ \\
\hline Negative & $7(26)$ \\
\hline \multicolumn{2}{|l|}{ Progesterone receptor } \\
\hline Positive & $20(74)$ \\
\hline Negative & $7(26)$ \\
\hline \multicolumn{2}{|l|}{ HER2 } \\
\hline Positive & $9(33)$ \\
\hline Negative & $18(67)$ \\
\hline \multicolumn{2}{|l|}{ Lymph Node Status } \\
\hline No & $12(44)$ \\
\hline $\mathrm{N} 1+$ & $15(56)$ \\
\hline \multicolumn{2}{|l|}{ T stage } \\
\hline I & $8(30)$ \\
\hline ॥ & $13(48)$ \\
\hline III & $4(15)$ \\
\hline IV & $2(7)$ \\
\hline \multicolumn{2}{|l|}{ Microvessel density } \\
\hline Low & $14(52)$ \\
\hline High & $13(48)$ \\
\hline
\end{tabular}

more rapid clearance of contrast as reflected by peak SER and WF on DCE-MRI. In an exploratory analysis, we found multiple promising radiomic features that may also predict MVD levels, with GLCM- ClusterProminence demonstrating the highest association. Our findings suggest that semi-quantitative DCE-MRI features correlate with tumor angiogenesis as measured by MVD and could potentially map to other molecular steps of the angiogenesis cascade 22 . In this way, semi-quantitative DCE-MRI offers the potential for multiparametric, simultaneous, and noninvasive assessment of the angiogenic process in its native context. Our results may also be translatable to clinical practice as our study utilized a standard ACR accredited clinical MRI, which emphasizes spatial rather than temporal resolution as with many research protocols. WF in particular, is routinely reported on commercially available computer-aided evaluation software tools (e.g. CADstream, Merge Healthcare Inc, Chicago, IL) allowing for straightforward implementation in clinical practice.

Extending on the clinical implications of these findings, semi-quantitative DCE-MRI could hold additional prognostic potential, particularly in the neoadjuvant setting. In principle, 

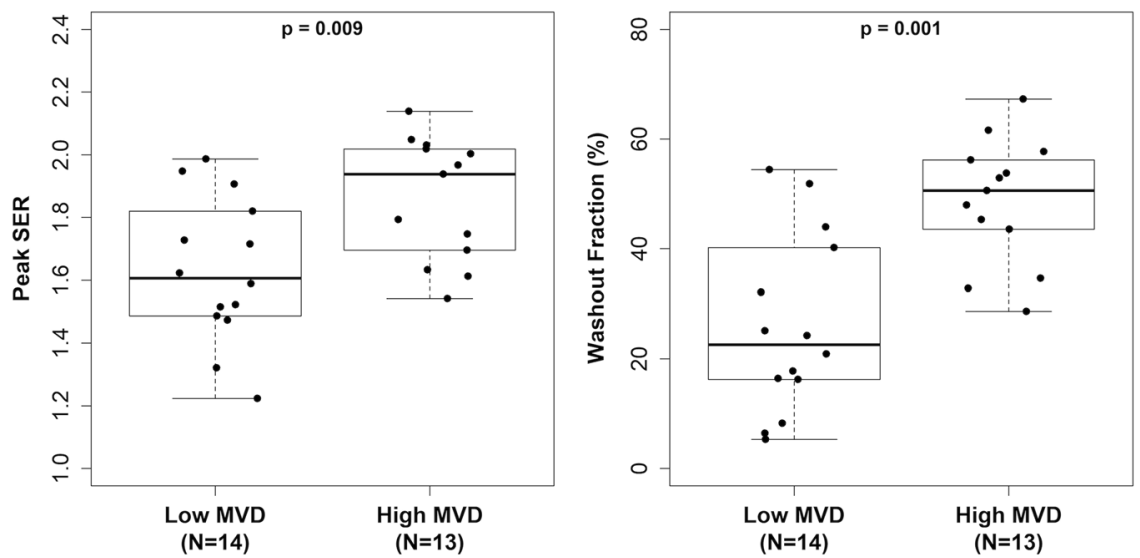

Fig. 1 DCE-MRI kinetics features associated with microvessel density. Two kinetics features significantly differentiated lesions with low versus high microvessel density (MVD). Box-and-whisker plots represent the median (solid line), inter-quartile range (IQR; box), and range of the dynamic contrast-enhanced (DCE)-MRI metrics. The whiskers extend up to 1.5 times the IQR from the box to the smallest and largest points. Lesions with higher MVD based on CD31 staining on average demonstrated higher peak signal enhancement ratio (SER; AUC: $0.79, p=$ 0.009 , left) and higher washout fraction (AUC: $0.87, p=0.001$, right).

Table 2. Univariable associations of kinetic parameters with MVD.

\begin{tabular}{|c|c|c|c|c|c|c|c|}
\hline Variable & $\begin{array}{l}\text { Low } \\
(N=14)\end{array}$ & $\begin{array}{l}\text { High } \\
(N=13)\end{array}$ & $O R^{b}$ & $(95 \% \mathrm{Cl})$ & $A \cup C$ & $(95 \% \mathrm{Cl})$ & $P$ \\
\hline Peak PE (\%) & $199(133,258)$ & $214(106,332)$ & 1.4 & $(0.6,3.2)$ & 0.61 & $(0.38,0.84)$ & 0.35 \\
\hline Peak SER & $1.61(1.22,1.99)$ & $1.94(1.54,2.14)$ & 3.3 & $(1.2,9.2)$ & 0.79 & $(0.62,0.96)$ & 0.009 \\
\hline $\mathrm{FTV}\left(\mathrm{cm}^{3}\right)$ & $4.7(0.4,93.6)$ & $3.0(0.3,85.2)$ & 0.8 & $(0.4,1.9)$ & 0.60 & $(0.38,0.82)$ & 0.40 \\
\hline
\end{tabular}

anti-angiogenic therapy could limit tumor growth and normalize vasculature, thereby improving delivery of neoadjuvant therapy ${ }^{22-24}$. However, clinical trials conducted over the last decade in unselected breast cancer patients have not shown increased overall survival with the addition of anti-angiogenic therapy, despite increased $\mathrm{pCR}$ in some studies ${ }^{2,25-27}$. At present, there are parallel efforts to identify biologically based high-risk subgroups benefiting from anti-angiogenic targeting, including inflammatory breast cancers ${ }^{28}$, metaplastic subtype ${ }^{29}$, and in triple negative breast cancers in BRCA1/2 mutation carriers ${ }^{30}$. In addition, there is sustained interest in combining antiangiogenesis agents with immune checkpoint blockade ${ }^{31-33}$. Vascular normalization could have particular relevance in the triple negative breast cancer setting where the addition of pembrolizumab to neoadjuvant chemotherapy has been shown to increase pathologic complete response $\mathrm{s}^{34}$. Our study suggests that semi-quantitative DCE-MRI could provide a non-invasive method for querying the angiogenic microenvironment in these clinically important subgroups, and could complement other molecular approaches for biomarker development including circulating growth factors, metabolic proteins, and hypoxia molecular signatures ${ }^{35-38}$.

Previous studies exploring the association with DCE-MRI and MVD have demonstrated somewhat variable results ${ }^{39-45}$. Similar to our findings, Esserman et al found in their study of 32 patients that individual SER values were significantly correlated with intratumoral MVD hot spots ${ }^{39}$. Poellinger et al also demonstrated positive associations with early contrast enhancement (initial peak $\mathrm{PE})$ and $M V D^{40}$. However, multiple other studies did not find a correlation with MVD and DCE-MRI kinetic features, although these studies differed significantly in their technique, most notably with regards to MRI acquisition protocols ${ }^{16,41-43}$. A recent investigation by Mori et al specifically focused on utilization of an alternative ultrafast DCE-MRI protocol to assess enhancement kinetics ${ }^{41}$. Interestingly, they found correlation of several ultrafast semi-quantitative kinetic parameters with MVD, most notably initial slope of enhancement, but did not find any such correlation using standard DCE timings and parameters including SER. Ultimately, the ability of kinetics-based measures to serve as meaningful biomarkers may be limited by varying technical approaches specific to each site, and are therefore likely to be less robust and translatable.

Radiomics analysis has been touted to circumvent the issue of inter-platform variability and may offer more reproducible results compared to semi-quantitative and/or quantitative kinetic features. This is because radiomics features can be extracted utilizing a standardized computational approach ${ }^{46}$ based on data acquired at a single timepoint, whereas traditional kinetic features rely heavily on arterial input functions and imaging timings, which may contribute to conflicting results such as those discussed above. Multiple early studies have demonstrated promise for radiomics features to correlate with prognostic factors of breast cancers, including treatment response, genomic assays of recurrence risk, and level of immune activation ${ }^{47-51}$. Few limited 


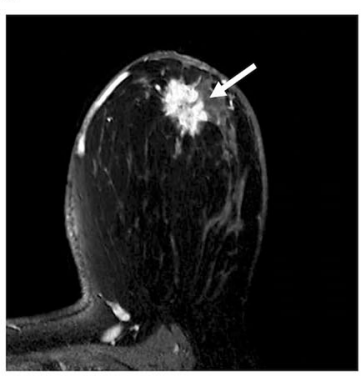

e

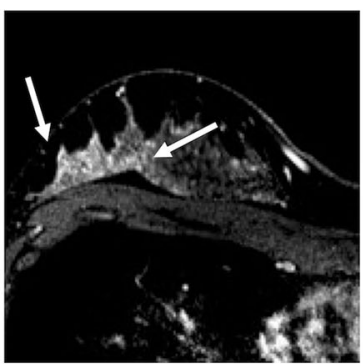

b

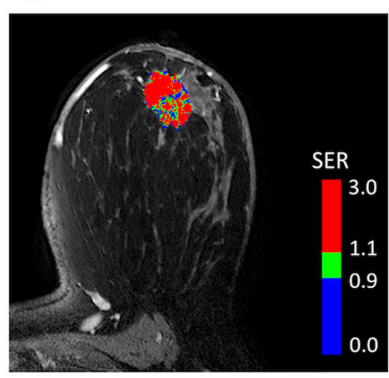

f

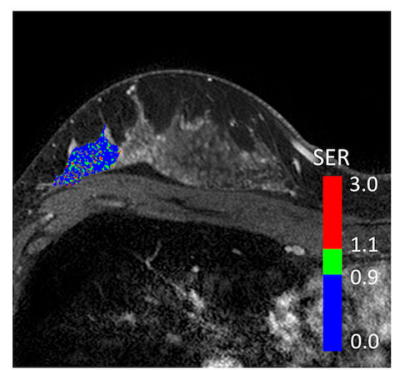

C

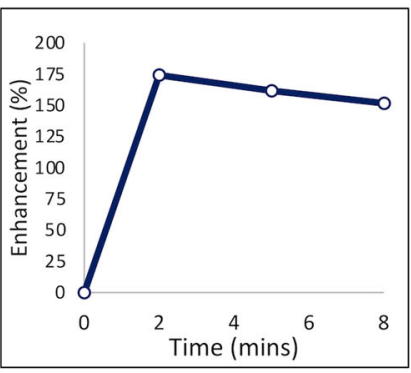

g

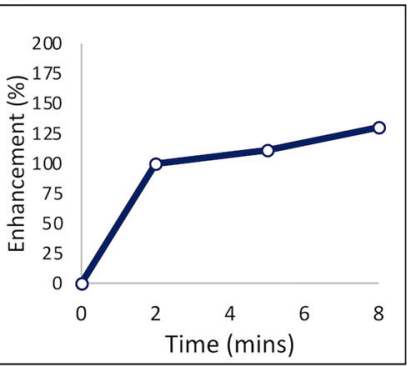

d

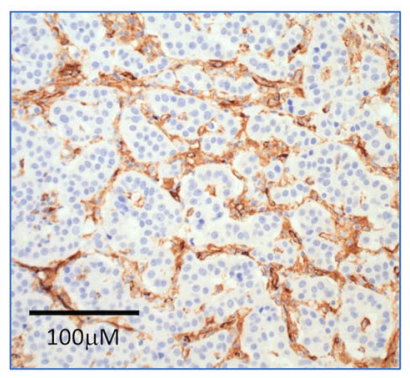

h

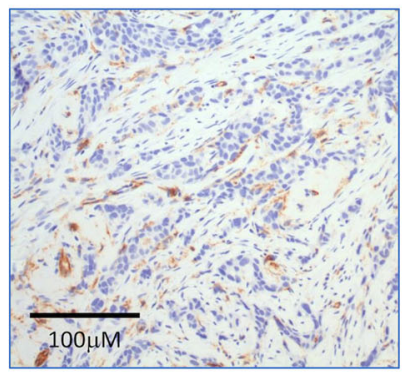

Fig. 2 DCE-MRI characteristics in two malignancies with different levels of microvessel density. Top row: a 44-year old woman with biopsyproven grade II invasive ductal carcinoma. a DCE-MRI demonstrates an enhancing lesion in the subareolar region of the left breast (arrow). b Kinetics analysis shows rapid contrast enhancement and washout, with predominantly high signal enhancement ratio (SER), with peak $\mathrm{SER}=2.1$ (initial peak percent enhancement $[\mathrm{PE}]=310 \%$, functional tumor volume $[\mathrm{FTV}]=4.9 \mathrm{~cm}^{3}$, and washout fraction $[\mathrm{WF}]=56 \%$; red indicates areas of washout [SER $\geq 1.1]$ ). c Plot of overall lesion enhancement (\%) versus time after contrast injection demonstrates high early peak enhancement and washout by $8 \mathrm{~min}$. d Histologic staining for CD31 indicates high microvessel density (MVD; shown at $\times 400$ magnification), with mean vessel count $=56$. Bottom row: a 34-year old woman with biopsy-proven grade III invasive ductal carcinoma. e DCEMRI demonstrates an enhancing lesion in the posterior right breast. $\mathbf{f}$ Kinetics analysis shows predominantly low SER, with peak SER $=1.3$ (initial peak $\mathrm{PE}=218 \%, \mathrm{FTV}=4.8 \mathrm{~cm}^{3}$, and $\left.\mathrm{WF}=6 \%\right)$ ), with mean vessel count $=9$. g Plot of overall lesion enhancement (\%) versus time after contrast injection demonstrates persistent enhancement over $8 \mathrm{~min}$. $\mathbf{h}$ Histologic staining for CD31 indicates low MVD (shown at $\times 400$ magnification).

Table 3. Exploratory univariable analysis of associations between MVD and selected ${ }^{\mathrm{a}}$ DCE-MRI radiomic texture features.

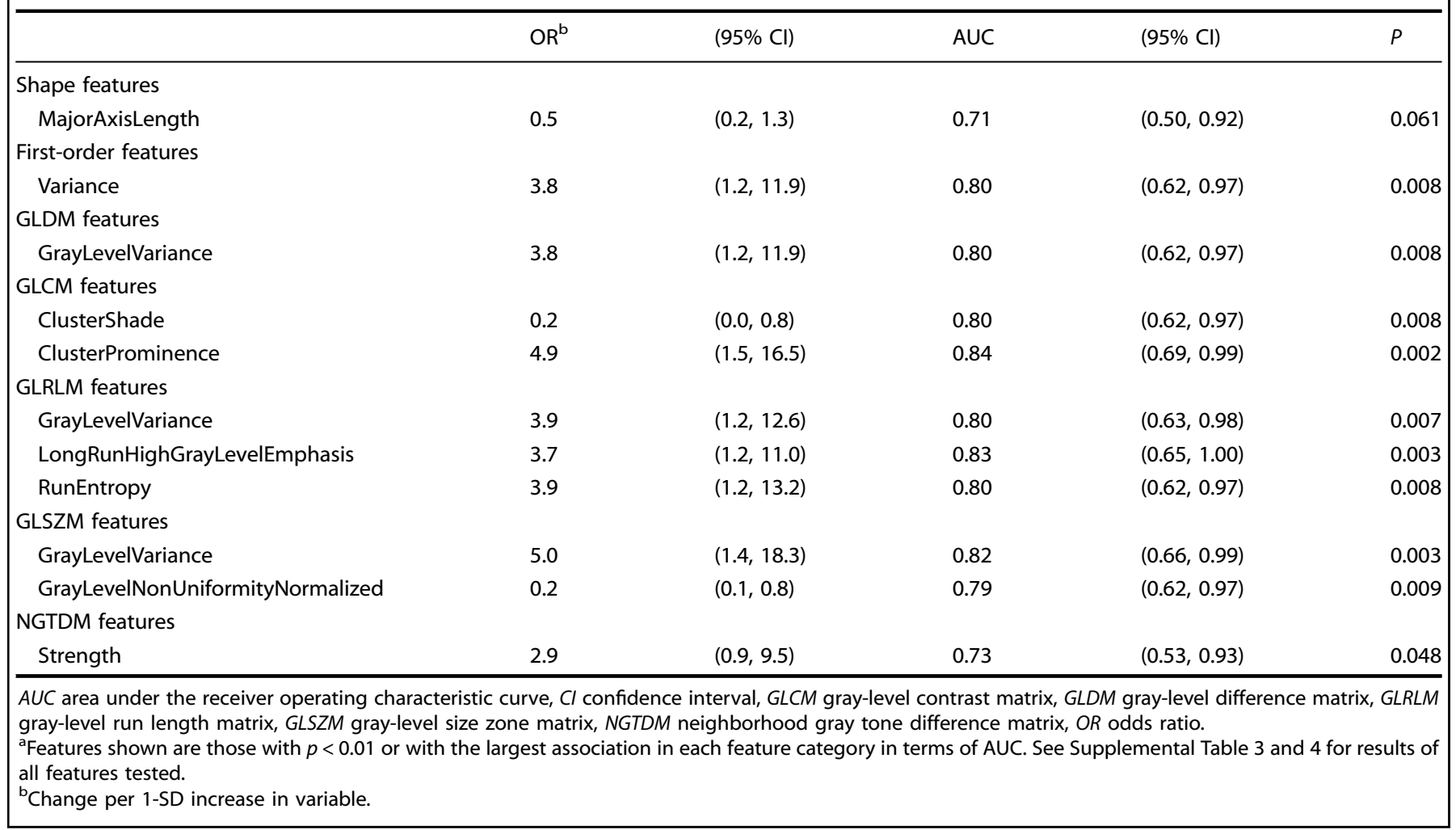



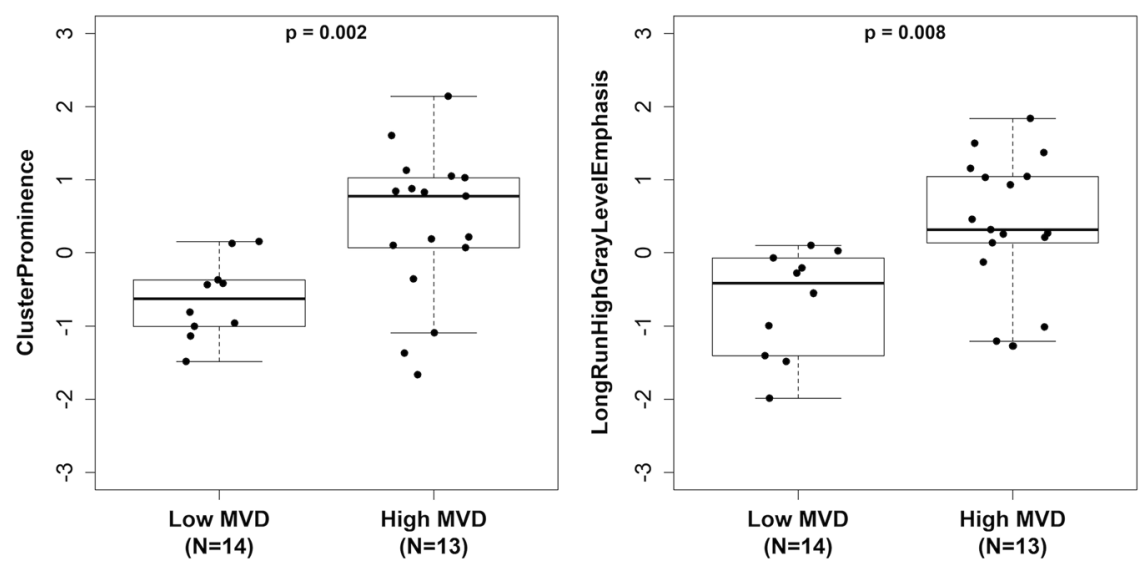

Fig. 3 DCE-MRI radiomics features most associated with microvessel density. Two radiomic texture features that significantly differentiated lesions with low versus high microvessel density (MVD). Box-and-whisker plots represent the median (solid line), inter-quartile range (IQR; box) and range of the radiomics features. The whiskers extend up to 1.5 times the IQR from the box to the smallest and largest points. Radiomics features were log-transformed to reduce skewness and standardized to have a standard deviation equal to 1. Lesions with higher MVD based on CD31 staining on average demonstrated higher GLCM-ClusterProminence values (AUC: $0.84, p=0.002$, left) and higher GLRLMLongRunHighGrayLevelEmphasis values (AUC: 0.83, $p=0.003$, right).

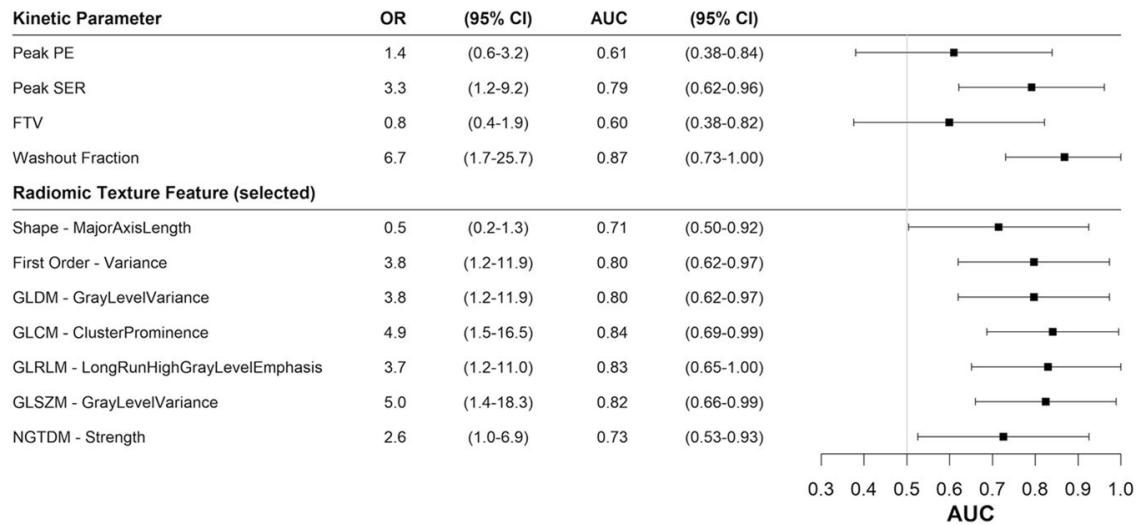

Fig. 4 Performance of DCE-MRI kinetics and radiomics features for distinguishing lesions with different microvessel densities. Odds ratios (ORs) and forest plot of area under the receiver operating characteristic curve (AUC) for predicting high microvascular density (MVD) are shown for all kinetic parameters and selected radiomic texture features. ORs are scaled to correspond to differences per 1-standard deviation increase. The error bars represent $95 \%$ confidence intervals (Cls). The texture feature with the strongest association with MVD in terms of AUC is shown for each radiomic feature category. See Table 3 and Supplementary Table 2 for more detail.

studies have explored associations of radiomics features with $M V D$, and to our knowledge only in non-breast malignancies, such as prostate cancer and renal cell carcinoma. These studies demonstrated promising results with several gray level and wavelet features correlating with $\mathrm{MVD}^{52,53}$. Our study found that of radiomic features, GLCM and GLRLM features, which primarily reflect spatial coherence and connectivity between voxels, most significantly correlated with MVD in breast cancers. Similarly, Yin et al also found several GLRLM features correlating with MVD in renal cell carcinomas ${ }^{53}$. Future work is needed to determine whether these relationships between radiomics features and MVD or other tumor angiogenesis markers persist in larger studies and across tumor types.

Another potential explanation for varying results between studies is variation in MVD assessment. Although most studies including our study used a similar technique by counting microvessels at the highest areas of neovascularization, there is no universally recommended immunostaining for MVD. In our study, MVD was measured using immunostaining with CD31, whereas several other studies utilized either factor VIII antigen or CD34. Both CD31 and CD34 have been shown to be more sensitive compared to factor VIII antigen in the assessment of MVD due to staining of isolated endothelial cells and immature vessels in addition to larger microvessels ${ }^{54}$. However, CD31 can react mildly with inflammatory cells and is rarely expressed strongly, with staining failure rates of up to $20 \%{ }^{4,45}$. Some studies suggest CD34 may be superior to CD31 as it is more strongly expressed on microvessels ${ }^{4,54}$.

Higher levels of MVD have been shown to predict poor survival and correlate with aggressive subtypes of breast cancer, such as higher histologic grade and negative estrogen receptor status $^{4,11,12}$. However, MVD assessment is known to have inter-reader variability, with several different studies attempting to minimize its subjectivity ${ }^{4,14}$. In prior studies, MVD is most commonly assessed as a binary variable, with many studies using the median value as the cutoff ${ }^{4}$. Interestingly, there are no standard published cutoff values for high MVD. In our study, we therefore assessed MVD as both a continuous and categorical variable.

While our study demonstrated a correlation of higher peak SER and WF with increased MVD, we did not identify initial peak PE and FTV to be similarly related to MVD. This likely results from the fact that early tumor enhancement in the first several minutes 
(from which both initial peak PE and FTV metrics are calculated) is determined by multiple additional factors beyond MVD. Specifically, initial peak PE is believed to be affected by blood flow, in addition to technical aspects such as contrast dose and injection rate $^{55}$, and other patient factors such as cardiac function. Furthermore, FTV is also primarily weighted by tumor size and may be less likely to demonstrate a direct relationship with MVD. In contrast, SER and WF are metrics that both reflect the degree of washout from the tumor, which we now posit is more directly related to the density of leaky and abnormal vessels that have been recruited to supply an aggressive malignancy and are less dependent on other factors.

Our study has several important limitations. First, we utilized CD31 for immunostaining of MVD, which as noted above may be less sensitive when compared to CD34. Second, our study did not include benign lesions for comparison, and thus we cannot directly determine that imaging features' associations with MVD have clear diagnostic value. Third, our sample size was relatively small, which limited our ability to assess the relative value of the different DCE-MRI radiomics metrics and perform multivariable analyses. Fourth, our study explored semi-quantitative rather than quantitative kinetic parameters. Quantitative measurements such as Ktrans, Vp, and Kep were not included because our study used clinical DCE-MRI scans, which emphasizes spatial resolution over temporal resolution and does not allow for pharmacokinetic mapping. Most pharmacokinetic mapping breast MRI studies sacrifice coverage or spatial resolution for rapid temporal sampling and are not as amenable for clinical interpretation or radiomics analysis. Finally, our analysis of texture features was hypothesis-generating and did not fully adjust for multiple comparisons. While some features appeared to have promising performance at discriminating between higher and lower MVD, these findings should be interpreted with caution and further studies in larger cohorts are warranted.

In conclusion, our study demonstrates that semi-quantitative DCE-MRI features have promise to serve as markers of MVD in invasive breast cancers, and could potentially identify additional non-invasive features mapping to other molecular steps of the angiogenesis cascade. Furthermore, our findings suggest that radiomics features may provide an even more robust approach to non-invasively evaluating MVD. If validated in larger studies, these features could provide prognostic value and aid in identifying patient subgroups who may benefit from anti-angiogenic therapy and also complement other molecular approaches for biomarker development.

\section{METHODS}

\section{Patients}

This prospective study was approved by the Institutional Review Board of the Fred Hutchinson Cancer Research Center (IR\#8148) and was compliant with the Health Insurance Portability and Accountability Act. All patients provided informed consent and were enrolled at the University of Washington/Seattle Cancer Care Alliance (SCCA) between January 2016 and July 2017. Patients over the age of 18 were eligible for the study if they had a new diagnosis of stage I - III invasive breast cancer with a minimum lesion size of $1.5 \mathrm{~cm}$ identified on core needle biopsy and underwent a clinical breast MRI at SCCA to evaluate the extent of disease. Patients were not eligible if they were treated with chemotherapy before the MRI or surgical treatment of the cancer, as this could affect both the MRI and pathology features measured.

\section{MRI Acquisition}

The MRI protocols followed guidelines established by the American College of Radiology breast MRI accreditation program ${ }^{56}$. The protocol included a 3D T1-weighted fast gradient echo-based DCE series with one pre and three sequential post-gadolinium contrast-enhanced sequences with scan durations of approximately $3 \mathrm{~min}$ each. All scans were performed on a 3 Tesla Philips Achieva Tx system (Philips Medical Systems, Best, the Netherlands) with a 16-channel breast coil (Mammotrak, Philips Healthcare). DCE-MRI was performed using a fat saturated 3D fast gradient echo sequence (eTHRIVE: enhanced T1-weighted High Resolution Isotropic Volume Excitation) with the following parameters: $T R / T E=5.9 / 3 \mathrm{msec}$, flip angle $=10^{\circ}, \quad$ spatial resolution $=0.5 \times 0.5 \times 1.3 \mathrm{~mm}$. Post-contrast sequences were acquired with $\mathrm{k}$ space centered at approximately 120 , 300 , and $480 \mathrm{~s}$ after contrast injection. All scans were acquired in the axial orientation. The contrast agent administered was $0.1 \mathrm{mmol} / \mathrm{kg}$ body weight gadoteridol (ProHance, Bracco Diagnostics, Milan, Italy) at the rate of $2 \mathrm{cc} / \mathrm{s}$ followed by a $15 \mathrm{cc}$ saline flush.

\section{DCE-MRI image analysis}

All MR image analyses were performed with observers blinded to pathologic outcomes, using customized open source software tools for kinetic enhancement measurements and radiomic texture measurements as detailed below.

Kinetic Analysis. DCE-MRI imaging volumes were first co-registered using a commercially available computer-aided evaluation software tool (CADstream, Merge Healthcare Inc, Chicago, IL) to correct for any misregistration due to patient motion between pre and post-contrast acquisitions. Next, semiautomated tumor segmentation and quantitation were performed by imaging researchers (N.S., M.H.) under the guidance of a radiologist (H.R., 10 years experience breast imaging) and imaging scientist (S.C.P., 15 years experience in quantitative breast MRI) who reviewed all tumor segmentations. Tumors were segmented on DCE-MRI using a custom software tool developed in MATLAB (MathWorks, Natick, MA) to select enhancing voxels and define a $3 D$ region of interest. Kinetics analysis was then performed using semiautomated software written in-house in Java language and ImageJ ( $\mathrm{NIH}$, public domain, Bethesda, MD). Contrast kinetics were characterized by several parameters as previous described ${ }^{57,58}$ : (1) Initial PE, (2) SER, (3) FTV, and (4) WF. Initial PE (units, \%) reflects the degree of signal enhancement in the tumor at $120 \mathrm{~s}$ after contrast delivery, calculated by

$\mathrm{PE}=\frac{S_{1}-S_{0}}{S_{0}} \times 100$

where $S_{0}$ is the MRI signal intensity prior to contrast and $S_{1}$ is the MRI signal intensity $120 \mathrm{~s}$ after contrast delivery. SER is a unitless index that reflects the rate of contrast washout in the tumor between 120 and $480 \mathrm{~s}$ after contrast delivery, calculated by:

$\mathrm{SER}=\frac{S_{1}-S_{0}}{S_{2}-S_{0}}$

where $S_{2}$ is the MRI signal intensity at $480 \mathrm{~s}$ after contrast delivery. Initial $\mathrm{PE}$ and SER were calculated on a voxel-by-voxel basis within the tumor, with SER calculated only for voxels with at least $50 \%$ initial PE. To determine peak PE and peak SER, the software automatically identified independent hot-spot tumor regions of $3 \times 3 \times 3$ voxels $\left(0.1625 \mathrm{~mm}^{3}\right)$ producing the highest mean initial PE and SER value, respectively. FTV (units, $\mathrm{cm}^{3}$ ) was calculated by summing volumes for all voxels with initial $P E \geq 50 \%$. Additionally, WF percentage was calculated as the fraction of all tumor voxels exhibiting washout (defined as those with $\mathrm{PE} \geq 50 \%$ and SER $\geq 1.1$.

Radiomic analysis. The 3D segmented lesions were also evaluated using an open source software platform for radiomics analyses (3D Slicer v4.11.0 with pyradiomics library ${ }^{59}$, www.slicer.org) of the initial contrast-enhanced DCE-MR images (120 s post-contrast). Each DCE-MRI dataset (volumetric stack) was normalized to have zero mean and unit variance, and isotropic resampling in 3 dimensions was applied before the radiomics features were calculated. A total of 108 standardized radiomics features ${ }^{46}$ were generated on each tumor volume for exploratory statistical analysis, within the following feature categories: (1) first-order histogram statistics based on voxel signal intensities (gray levels), (2) shape-based descriptors, (3) gray level co-occurrence matrix (GLCM) based on the second-order joint probability functions of voxel intensities in a particular spatial region, (4) gray level size zone matrix (GLSZM), quantifying gray level zones as number of connected voxels with the same gray level intensity, (5) gray level run length matrix (GLRLM), quantifying gray level runs as length (number of consecutive pixels) with the same gray level value, (6) gray level dependence matrix (GLDM), quantifying the number of connected voxels in a given distance that are dependent on the center voxel, and (7) neighboring gray tone difference matrix (NGTDM), quantifying differences 


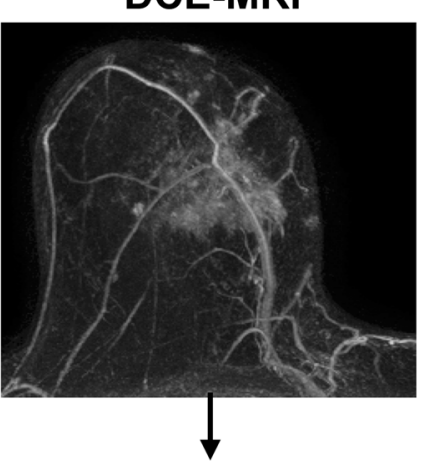

\section{D Segmentation}

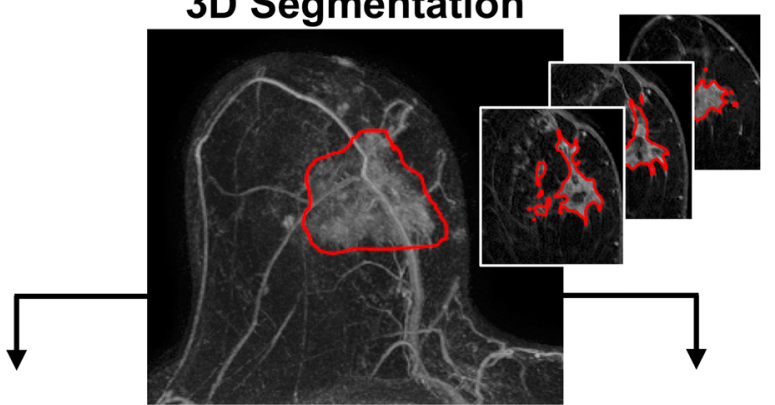

Kinetics

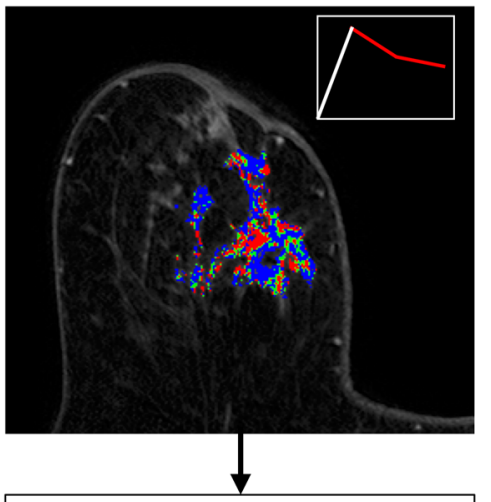

Kinetic Parameters

- Peak PE

- Peak SER

- FTV

- Washout Fraction
Radiomics

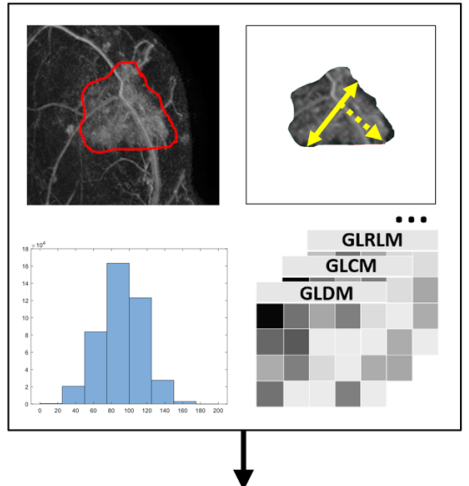

Shape, First Order, Texture Features

- GLCM

- GLRLM

- GLDM

- GLSZM

- NGDTV

Fig. 5 Postprocessing pipeline for quantitative DCE-MRI lesion assessment. Both kinetics and radiomics parameters are extracted for threedimensional segmented tumor volumes (illustrated in 62-year old woman with biopsy proven invasive ductal carcinoma). DCE-MRI dynamic contrast-enhanced magnetic resonance imaging, PE percent enhancement, SER signal enhancement ratio, FTV functional tumor volume, GLCM gray-level contrast matrix, GLRLM gray-level run length matrix, GLDM gray-level difference matrix, GLSZM gray-level size zone matrix, NGTDM neighborhood gray tone difference matrix.

in gray levels of neighboring voxels. The MR imaging postprocessing pipeline is summarized in Fig. 5.

\section{Pathologic assessment}

All biopsy and surgical specimens pathologically assessed at our institution include verification of invasive cancer, tumor grade (by Nottingham histologic score reflecting tumor cell differentiation based on tubule formation, nuclear grade, and mitotic rate), ER and PR status (by Allred score for expression), HER2 status (positive or negative by immunohistochemistry and/or fluorescence in situ hybridization), and Ki-67 proliferation index. For the study, MVD was assessed by immunostaining with mouse antihuman $\mathrm{CD} 31$ monoclonal antibody $(\mathrm{JC} / 70 \mathrm{~A})$ on a single representative tumor specimen slide obtained from the surgical tissue block (or from the diagnostic core needle biopsy tissue block in patients who underwent chemotherapy treatment before their surgery). The two most vascularized areas within the tumor ('hot spots') were chosen at low magnification $(\times 40)$ and vessels were counted in a representative high 
magnification $\left(x 400 ; 0.152 \mathrm{~mm}^{2} ; 0.44 \mathrm{~mm}\right.$ diameter) field in each of these two areas. Single immunoreactive endothelial cells, or endothelial cell clusters separate from other microvessels, were counted as individual microvessels. Endothelial staining in large vessels with tunica media, and nonspecific staining of nonendothelial structures, were disregarded in microvessel counts. The MVD counts from the two microscopic fields per specimen were averaged. Two board certified anatomic pathologists specializing in breast pathology (M.H.R. 10 years, E.U.P. two years subspecialty experience) independently reviewed the same CD31-stained slides to assess MVD as described, blinded to the clinical and imaging data. MVD counts from the two pathologists were averaged for the statistical analysis, except those where the difference was large $(\geq 12)$, which were adjudicated by reviewing the case together to reach consensus.

\section{Statistical analyses}

Continuous variables were summarized as median (range) and categorical variables as number (percent). For the primary analysis, univariable associations were assessed between kinetic parameters and MVD dichotomized as low ( $\leq$ median value) vs. high ( $>$ median value). Associations were summarized using odds ratios (ORs) from univariable logistic regression models and the area under the receiver operating characteristic curve (AUC). Differences between lower and higher MVD were tested using the Wilcoxon rank-sum test. Due to testing four kinetic parameters, we used a Bonferroni-corrected significance threshold of $a=$ 0.0125 to determine statistical significance. As a sensitivity analysis, we also performed an analogous analysis where we used Spearman's rank correlation coefficient to evaluate associations of kinetic parameters with MVD as a continuous variable.

As a secondary, hypothesis-generating analysis, we used similar methods to evaluate associations of radiomic texture features with MVD, both as a dichotomous variable and a continuous variable. We used a significance threshold $a=0.01$ to reduce the risk of false positive findings, though did not perform a full adjustment for multiple comparisons to avoid a substantial loss of statistical power. Two-sided confidence intervals (Cls) were calculated for all ORs, AUC values, and Spearman's rank correlation coefficients. A confidence level of $95 \%$ was used for all Cls. All statistical tests performed were two-sided. Statistical calculations were conducted with the statistical computing language $\mathrm{R}$ (version 3.1.1; R Foundation for Statistical Computing, Vienna, Austria).

\section{Reporting summary}

Further information on research design is available in the Nature Research Reporting Summary linked to this article.

\section{DATA AVAILABILITY}

The data generated and analyzed during this study are described in the following figshare data record: https://doi.org/10.6084/m9.figshare.13574570 ${ }^{60}$. The deidentified DCE-MRI data, measures and metadata will be shared as part of the figshare data record. Deidentified MR images will be available upon request, and interested parties should contact the corresponding author. The pathology CD-31 stained slides data are not publicly available for the following reason: no digitized versions of the slides have been created.

\section{CODE AVAILABILITY}

The open source software platform 3D Slicer (v4.11.0 with pyradiomics library, www. slicer.org) was utilized for radiomics analysis as referenced in the methods section. Software for lesion segmentation and kinetics analysis were developed in-house using MATLAB (MathWorks, Natick, MA) and the open source software platform ImageJ (NIH, public domain, Bethesda, MD), respectively, and can be made available upon request to the corresponding author.

Received: 15 April 2020; Accepted: 15 March 2021; Published online: 16 April 2021

\section{REFERENCES}

1. Tower, H., Ruppert, M. \& Britt, K. The immune microenvironment of breast cancer progression. Cancers 11, 1375 (2019).
2. Aalders, K. C., Tryfonidis, K., Senkus, E. \& Cardoso, F. Anti-angiogenic treatment in breast cancer: facts, successes, failures and future perspectives. Cancer Treat. Rev. 53, 98-110 (2017).

3. Ferrara, N. \& Davis-Smyth, T. The biology of vascular endothelial growth factor. Endocr. Rev. 18, 4-25 (1997).

4. Uzzan, B., Nicolas, P., Cucherat, M. \& Perret, G.-Y. Microvessel density as a prognostic factor in women with breast cancer: a systematic review of the literature and meta-analysis. Cancer Res. 64, 2941-2955 (2004).

5. Kim, S.-W. et al. Identification of angiogenesis in primary breast carcinoma according to the image analysis. Breast Cancer Res. Treat. 74, 121-129 (2002).

6. Horak, E. R. et al. Angiogenesis, assessed by platelet/endothelial cell adhesion molecule antibodies, as indicator of node metastases and survival in breast cancer. Lancet 340, 1120-1124 (1992).

7. Hanahan, D. \& Weinberg, R. A. Hallmarks of cancer: the next generation. Cell 144, 646-674 (2011).

8. Longatto Filho, A., Lopes, J. M. \& Schmitt, F. C. Angiogenesis and breast cancer. J. Oncol. 2010, 576384 (2010).

9. Weidner, N., Semple, J. P., Welch, W. R. \& Folkman, J. Tumor angiogenesis and metastasis-correlation in invasive breast carcinoma. N. Engl. J. Med. 324, 1-8 (1991).

10. Şener, E., Şipal, S. \& Gündoğdu, C. Comparison of microvessel density with prognostic factors in invasive ductal carcinomas of the breast. Turk. Patoloji Derg. 32, 164-170 (2016).

11. Bujor, I. S. et al. Evaluation of vascular proliferation in molecular subtypes of breast cancer. Vivo 32, 79-83 (2018).

12. Arnes, J. B. et al. Vascular proliferation is a prognostic factor in breast cancer. Breast Cancer Res. Treat. 133, 501-510 (2012).

13. Sun, $C$. et al. Tumor angiogenesis and bone metastasis-correlation in invasive breast carcinoma. J. Immunol. Methods 452, 46-52 (2018).

14. Marien, K. M. et al. Development and validation of a histological method to measure microvessel density in whole-slide images of cancer tissue. PloS ONE 11, e0161496 (2016).

15. Hulka, C. A. et al. Benign and malignant breast lesions: differentiation with echoplanar MR imaging. Radiology 197, 33-38 (1995).

16. Knopp, M. V. et al. Pathophysiologic basis of contrast enhancement in breast tumors. J. Magn. Reson. Imaging JMRI 10, 260-266 (1999).

17. Mori, N. et al. Fast temporal resolution dynamic contrast-enhanced MRI: histogram analysis versus visual analysis for differentiating benign and malignant breast lesions. AJR Am. J. Roentgenol. 211, 933-939 (2018).

18. Weidner, N. The importance of tumor angiogenesis: the evidence continues to grow. Am. J. Clin. Pathol. 122, 675-677 (2004).

19. Buadu, L. D. et al. Breast lesions: correlation of contrast medium enhancement patterns on MR images with histopathologic findings and tumor angiogenesis. Radiology 200, 639-649 (1996).

20. Gillies, R. J., Kinahan, P. E. \& Hricak, H. Radiomics: images are more than pictures, they are data. Radiology 278, 563-577 (2016).

21. Saltz, J. et al. Towards generation, management, and exploration of combined radiomics and pathomics datasets for cancer research. AMIA Jt. Summits Transl. Sci. 2017, 85-94 (2017).

22. De Palma, M., Biziato, D. \& Petrova, T. V. Microenvironmental regulation of tumour angiogenesis. Nat. Rev. Cancer 17, 457-474 (2017).

23. Folkman, J. Tumor angiogenesis: therapeutic implications. N. Engl. J. Med. 285, 1182-1186 (1971).

24. Potente, M., Gerhardt, H. \& Carmeliet, P. Basic and therapeutic aspects of angiogenesis. Cell 146, 873-887 (2011).

25. Bear, H. D. et al. Bevacizumab added to neoadjuvant chemotherapy for breast cancer. N. Engl. J. Med. 366, 310-320 (2012).

26. von Minckwitz, G. et al. Neoadjuvant chemotherapy and bevacizumab for HER2negative breast cancer. N. Engl. J. Med. 366, 299-309 (2012).

27. Miller, K. D. et al. Double-blind phase III trial of adjuvant chemotherapy with and without bevacizumab in patients with lymph node-positive and high-risk lymph node-negative breast cancer (E5103). J. Clin. Oncol. 36, 2621-2629 (2018).

28. Pierga, J.-Y. et al. Pathological response and circulating tumor cell count identifies treated HER2 + inflammatory breast cancer patients with excellent prognosis: BEVERLY-2 survival data. Clin. Cancer Res. 21, 1298-1304 (2015).

29. Basho, R. K. et al. Targeting the PI3K/AKT/mTOR pathway for the treatment of mesenchymal triple-negative breast cancer: evidence from a phase 1 trial of mTor inhibition in combination with liposomal doxorubicin and bevacizumab. JAMA Oncol. 3, 509-515 (2017).

30. Fasching, P. A. et al. BRCA1/2 mutations and bevacizumab in the neoadjuvant treatment of breast cancer: response and prognosis results in patients with triplenegative breast cancer from the GeparQuinto Study. J. Clin. Oncol. 36, 2281-2287 (2018).

31. Liu, J. F. et al. Assessment of combined nivolumab and bevacizumab in relapsed ovarian cancer: a phase 2 clinical trial. JAMA Oncol. 5, 1731-1738 (2019). 
32. Hodi, F. S. et al. Bevacizumab plus ipilimumab in patients with metastatic melanoma. Cancer Immunol. Res. 2, 632-642 (2014).

33. Wallin, J. J. et al. Atezolizumab in combination with bevacizumab enhances antigen-specific T-cell migration in metastatic renal cell carcinoma. Nat. Commun. 7, 12624 (2016).

34. Schmid, P. et al. Pembrolizumab for early triple-negative breast cancer. N. Engl. J. Med. 382, 810-821 (2020).

35. Gianni, L. et al. Follow-up results of $\mathrm{NOAH}$, a randomized phase III trial evaluating neoadjuvant chemotherapy with trastuzumab $(\mathrm{CT}+\mathrm{H})$ followed by adjuvant $\mathrm{H}$ versus $C T$ alone, in patients with HER2-positive locally advanced breast cancer. J. Clin. Oncol. 31, 503-503 (2013).

36. Cameron, D. et al. Adjuvant bevacizumab-containing therapy in triple-negative breast cancer (BEATRICE): primary results of a randomised, phase 3 trial. Lancet Oncol. 14, 933-942 (2013).

37. Miles, D. et al. Bevacizumab plus paclitaxel versus placebo plus paclitaxel as firstline therapy for HER2-negative metastatic breast cancer (MERiDiAN): A doubleblind placebo-controlled randomised phase III trial with prospective biomarker evaluation. Eur. J. Cancer 70, 146-155 (2017).

38. Chihak, M. A. et al. Prognostic value of tumor response in pediatric and adult patients with Ewing sarcoma. J. Clin. Oncol. 34, 10543-10543 (2016).

39. Esserman, L., Hylton, N., George, T. \& Weidner, N. Contrast-enhanced magnetic resonance imaging to assess tumor histopathology and angiogenesis in breast carcinoma. Breast J. 5, 13-21 (1999).

40. Poellinger, A. et al. Correlation between enhancement characteristics of MR mammography and capillary density of breast lesions. Eur. J. Radiol. 83, 2129-2136 (2014).

41. Mori, N. et al. Ultrafast dynamic contrast-enhanced breast MRI: kinetic curve assessment using empirical mathematical model validated with histological microvessel density. Acad. Radiol. 26, e141-e149 (2019).

42. Su, M.-Y. et al. Correlation of dynamic contrast enhancement MRI parameters with microvessel density and VEGF for assessment of angiogenesis in breast cancer. J. Magn. Reson. Imaging 18, 467-477 (2003).

43. Teifke, A. et al. Dynamic MR imaging of breast lesions: correlation with microvessel distribution pattern and histologic characteristics of prognosis. Radiology 239, 351-360 (2006)

44. Kim, S. H. et al. Dynamic contrast-enhanced MRI perfusion parameters as imaging biomarkers of angiogenesis. PloS ONE 11, e0168632 (2016).

45. Li, L. et al. Parameters of dynamic contrast-enhanced MRI as imaging markers for angiogenesis and proliferation in human breast cancer. Med. Sci. Monit. 21, 376-382 (2015)

46. Zwanenburg, A. et al. The image biomarker standardization initiative: standardized quantitative radiomics for high-throughput image-based phenotyping. Radiology 295, 328-338 (2020).

47. $\mathrm{Li}, \mathrm{H}$. et al. MR imaging radiomics signatures for predicting the risk of breast cancer recurrence as given by research versions of MammaPrint, oncotype DX, and PAM50 gene assays. Radiology 281, 382-391 (2016).

48. Ashraf, A. B. et al. Identification of intrinsic imaging phenotypes for breast cancer tumors: preliminary associations with gene expression profiles. Radiology 272, 374-384 (2014)

49. Wu, J. et al. Magnetic resonance imaging and molecular features associated with tumor-infiltrating lymphocytes in breast cancer. Breast Cancer Res. 20, 101 (2018).

50. Braman, N. et al. Association of peritumoral radiomics with tumor biology and pathologic response to preoperative targeted therapy for HER2 (ERBB2)-positive breast cancer. JAMA Netw. Open 2, e192561 (2019).

51. Jahani, N. et al. Prediction of treatment response to neoadjuvant chemotherapy for breast cancer via early changes in tumor heterogeneity captured by DCE-MRI registration. Sci. Rep. 9, 12114 (2019).

52. Tanadini-Lang, S. et al. Exploratory radiomics in computed tomography perfusion of prostate cancer. Anticancer Res 38, 685-690 (2018).

53. Yin, Q. et al. Associations between tumor vascularity, vascular endothelial growth factor expression and PET/MRI radiomic signatures in primary clear-cell-renal-cellcarcinoma: proof-of-concept study. Sci. Rep. 7, 43356 (2017).

54. Martin, L. et al. Examining the technique of angiogenesis assessment in invasive breast cancer. Br. J. Cancer 76, 1046-1054 (1997).

55. van Niekerk, C. G. et al. Correlation between dynamic contrast-enhanced MRI and quantitative histopathologic microvascular parameters in organ-confined prostate cancer. Eur. Radiol. 24, 2597-2605 (2014).
56. American College of Radiology Breast Accreditation Program: Modalities Available from: https://www.acraccreditation.org/modalities/mri (accessed 22 August 2020).

57. Partridge, S. C. et al. Accuracy of MR imaging for revealing residual breast cance in patients who have undergone neoadjuvant chemotherapy. AJR Am. J. Roentgenol. 179, 1193-1199 (2002).

58. Partridge, S. C. et al. Association between serial dynamic contrast-enhanced MRI and dynamic 18F-FDG PET measures in patients undergoing neoadjuvant chemotherapy for locally advanced breast cancer. J. Magn. Reson. Imaging 32, 1124-1131 (2010).

59. van Griethuysen, J. J. M. et al. Computational radiomics system to decode the radiographic phenotype. Cancer Res. 77, e104-e107 (2017).

60. Xiao, J. et al. Metadata record for the manuscript: Dynamic Contrast-Enhanced Breast MRI Features Correlate with Invasive Breast Cancer Angiogenesis. figshare https://doi.org/10.6084/m9.figshare.13574570 (2021).

\section{ACKNOWLEDGEMENTS}

This research was funded in part through the $\mathrm{NIH} / \mathrm{NCl}$ Cancer Center Support Grant P30 CA015704, NIH/NCl grant R01CA248192 (SCP), R01CA203883 (HR), and Department of Defense W81XWH-18-1-0098 (KJC) and The Safeway Foundation.

\section{AUTHOR CONTRIBUTIONS}

All authors made substantial contributions to the intellectual content of this work via conception, design, data acquisition, analysis, and/or interpretation, in addition to drafting and/or critical revision of the disclosure. All authors gave final approval and are accountable to all aspects of the work in ensuring that questions related to the accuracy or integrity of any part of the work are appropriately investigated and resolved.

\section{COMPETING INTERESTS}

S.C.P., H.R. report receiving commercial research grants from General Electric Healthcare and commercial research support from Philips Healthcare. D.H. reports receiving research support from GE Healthcare, Philips Healthcare, and Canon Medical Systems USA. No competing interests were disclosed by the other authors.

\section{ADDITIONAL INFORMATION}

Supplementary information The online version contains supplementary material available at https://doi.org/10.1038/s41523-021-00247-3.

Correspondence and requests for materials should be addressed to S.C.P.

Reprints and permission information is available at http://www.nature.com/ reprints

Publisher's note Springer Nature remains neutral with regard to jurisdictional claims in published maps and institutional affiliations.

Open Access This article is licensed under a Creative Common Attribution 4.0 International License, which permits use, sharing, adaptation, distribution and reproduction in any medium or format, as long as you give appropriate credit to the original author(s) and the source, provide a link to the Creative Commons license, and indicate if changes were made. The images or other third party material in this article are included in the article's Creative Commons license, unless indicated otherwise in a credit line to the material. If material is not included in the article's Creative Commons license and your intended use is not permitted by statutory regulation or exceeds the permitted use, you will need to obtain permission directly from the copyright holder. To view a copy of this license, visit http://creativecommons. org/licenses/by/4.0/.

(c) The Author(s) 2021 